\section{Facharztprüfung zur Erlangung des Schwerpunktes Neuropädiatrie zum Facharzttitel FMH Kinder- und Jugendmedizin}

Aufgrund des Weiterbildungsprogrammes, welches am 1. Juli 2001 in Kraft gesetzt wurde, ist die Teilnahme an der Facharztprüfung für diejenigen Kandidatinnen und Kandidaten Voraussetzung für die Erlangung des Schwerpunktes Neuropädiatrie, welche ihre Weiterbildung in Neuropädiatrie bis am 31. Dezember 1995 nicht abgeschlossen hatten. Es empfiehlt sich, die Facharztprüfung frühestens im letzten Jahr der reglementarischen Weiterbildung abzulegen (Art. 23 WBO).
Ort und Datum: werden nach Eingang der Anmeldungen, abhängig von gewünschter Prüfungssprache, festgelegt.

Prüfungsgebühr: Die Fachgesellschaft erhebt eine Prüfungsgebühr von Fr. 400.-.

Anmeldung: unter Beilage des Curriculums an: Prof. E. Boltshauser, Uni-Kinderklinik, Abteilung Neurologie, Steinwiesstrasse 75, 8032 Zürich, Tel. 0126673 30, Fax 012667163.

Anmeldefrist: 31. Mai 2002

\section{Prüfung zur Erlangung der Fähigkeitsausweise Elektro- enzephalographie (SGKN), Elektroneuromyographie (SGKN) und Zerebrovaskuläre Krank- heiten (SGKN)}

Die Programme zur Erlangung der Fähigkeitsausweise Elektroenzephalographie (SGKN), Elektroneuromyographie (SGKN) und Zerebrovaskuläre Krankheiten (SGKN) sind seit dem 1. Januar 2000 bzw. seit dem 1. Januar 2001 in Kraft. Neben den übrigen Voraussetzungen, die im Internet unter http://www.fmh.ch/fmh.cfm abrufbar sind, ist eine abschliessende theoretische und praktische Prüfung vorgesehen. Die Prüfung kann frühestens ein Jahr vor dem voraussichtlichen Erwerb des Facharzttitels abgelegt werden. Kolleginnen und Kollegen, welche sich für die Prüfung anmelden, werden gebeten, die erforderlichen Unterlagen beizulegen.

Prüfungsdatum und -ort: Freitag, 8. November 2002, Kantonsspital, Basel.

Prüfungsgebühr:

Fr. 500.- Mitglieder der Schweizerischen Gesellschaft für klinische Neurophysiologie (SGKN) Fr. 1000.- Nicht-Mitglieder der SGKN

Anmeldung:

Prof. Dr. H. P. Ludin,

Flurhofstrasse 52, 9000 St. Gallen,

Tel. 07124443 70, Fax 0712444371.

Anmeldefrist: 30. Juni 2002 


\section{Examen de spécialiste en vue de l'obtention de la formation approfondie en neuropédiatrie à adjoindre au titre de spécialiste FMH en pédiatrie}

Selon le programme de formation postgraduée entré en vigueur le $1^{\text {er }}$ juillet 2001, la participation à l'examen de spécialiste est une condition requise pour les candidats à l'obtention de la formation approfondie en neuropédiatrie qui n'avaient pas terminé leur formation postgraduée en neuropédiatrie le 31 décembre 1995. Il leur est recommandé de passer l'examen de spécialiste au plus tôt durant la dernière année de leur formation postgraduée réglementaire (art. $23 \mathrm{RFP}$ ).
Lieu et date: Le lieu ainsi que la date de l'examen seront communiqués après réception des inscriptions et en fonction de la langue dans laquelle le candidat souhaite passer l'examen.

Taxe d'examen: La société de discipline médicale prélève une participation aux frais de Fr. 400.-.

Inscription: accompagnée de votre curriculum auprès du Prof. E. Boltshauser, Uni-Kinderklinik, Abteilung Neurologie, Steinwiesstrasse 75, 8032 Zurich, tél. 0126673 30, fax 012667163.

Délai d'inscription: le 31 mai 2002

\section{Examen pour l'attribution des certificats de formation complémentaire en électro- encéphalographie (SSNC), en électroneuromyographie (SSNC) et en maladies cérébro- vasculaires (SSNC)}

Les programmes menant à l'attribution des certificats de formation complémentaire en électroencéphalographie (SSNC), en électroneuromyographie (SSNC) et en maladies cérébrovasculaires (SSNC) sont entrés en vigueur le $1^{\text {er }}$ janvier 2000 et le $1^{\text {er }}$ janvier 2001 respectivement. On peut consulter sur Internet (http://www.fmh.ch/fmh.cfm) les conditions requises pour l'obtention de chacun des certificats. Une des conditions est la réussite d'un examen final, théorique et pratique. Ce n'est qu'une année au plus tôt avant l'acquisition probable du titre FMH de spécialiste que les candidats peuvent s'y présenter. Lors de leur inscription pour cet examen, les collègues sont priés de joindre les documents requis.

Date et lieu de l'examen: Vendredi 8 novembre 2002, Hôpital cantonal de Bâle.

Taxe d'examen:

Fr. 500.- Membres de la Société Suisse de Neurophysiologie Clinique (SSNC)

Fr. 1000.- Non-membres

Inscription:

Prof. Dr. H. P. Ludin,

Flurhofstrasse 52, 9000 St. Gallen,

tél. 07124443 70, fax 0712444371

Délai d'inscription: 30 juin 2002 